\title{
MEASUREMENT AND IMPLICATIONS OF THE COSMIC MICROWAVE BACKGROUND SPECTRUM
}

JOHN C. MATHER

Code 685, Laboratory for Astronomy and Solar Physics

NASA Goddard Space Flight Center, Greenbelt, MD 20771 $U S A$

\section{Abstract.}

The Cosmic Background Explorer (COBE) was developed by NASA Goddard Space Flight Center to measure the diffuse infrared and microwave radiation from the early universe. It also measured emission from nearby sources such as the stars, dust, molecules, atoms, ions, and electrons in the Milky Way, and dust and comets in the Solar System. It was launched 18 November 1989 on a Delta rocket, carrying one microwave instrument and two cryogenically cooled infrared instruments. The Far Infrared Absolute Spectrophotometer (FIRAS) mapped the sky at wavelengths from 0.01 to 1 $\mathrm{cm}$, and compared the CMBR to a precise blackbody. The spectrum of the CMBR differs from a blackbody by less than $0.03 \%$. The Differential Microwave Radiometers (DMR) measured the fluctuations in the CMBR originating in the Big Bang, with a total amplitude of 11 parts per million on a $10^{\circ}$ scale. These fluctuations are consistent with scale-invariant primordial fluctuations. The Diffuse Infrared Background Experiment (DIRBE) spanned the wavelength range from 1.2 to $240 \mu \mathrm{m}$ and mapped the sky at a wide range of solar elongation angles to distinguish foreground sources from a possible extragalactic Cosmic Infrared Background Radiation (CIBR). In this paper we summarize the COBE mission and describe the results from the FIRAS instrument. The results from the DMR and DIRBE were described by Smoot and Hauser at this Symposium. 


\section{COBE Concept}

The three COBE instruments cover the entire wavelength range from 1.2 $\mu \mathrm{m}$ to $1 \mathrm{~cm}$. The FIRAS (Far Infrared Absolute Spectrophotometer) spans $105 \mu \mathrm{m}$ to $1 \mathrm{~cm}$ in two bands with a $7^{\circ}$ beam. The DMR (Differential Microwave Radiometer) covers $31.5,53$, and $90 \mathrm{GHz}(9.5,5.6$, and $3 \mathrm{~mm})$, also with a $7^{\circ}$ beam. The DIRBE (Diffuse Infrared Background Experiment) spans 1.2 to $240 \mu \mathrm{m}$ with $10 \mathrm{bands}$, and also measures polarization in its three shorter bands. Previous works have described the COBE and early results (Mather et al. 1990; Mather et al. 1991a,b; Janssen and Gulkis 1991; Wright 1990, 1991; Hauser 1991a,b; Smoot et al. 1991; Smoot 1991; Bennett 1991; Boggess 1991; Mather 1982; Gulkis et al. 1990; Boggess 1992), and the engineering of the spacecraft has also been published (Barney 1991; Milam 1991; Hopkins and Castles 1985; Hopkins and Payne 1987; Volz and Ryschkewitsch 1990; Volz et al. 1990, 1991; Volz and DiPirro 1992; Mosier 1991; Coladonato et al. 1990; Sampler 1990; Bromberg and Croft 1985).

A liquid helium cryostat cooled the FIRAS and DIRBE to about 1.5 $\mathrm{K}$ for 10 months, and after the evaporation of the helium the temperature slowly rose to about $60 \mathrm{~K}$. The DMR radiometers are radiatively cooled, with the $31.5 \mathrm{GHz}$ receivers at $290 \mathrm{~K}$ and the others at $140 \mathrm{~K}$. A conical shield protects them from the Sun and Earth, which do not illuminate the instruments directly. The orbit is a sun-synchronous polar orbit, at 900 $\mathrm{km}$ altitude and $99^{\circ}$ inclination, with a $6 \mathrm{PM}$ ascending node. The Earth's gravitational quadrupole precesses the orbit plane to maintain a $6 \mathrm{PM}$ ascending node, so that the plane is always approximately perpendicular to the Sun. The spacecraft is oriented with its spin axis away from the Earth and about $94^{\circ}$ to the Sun. It spins at $0.8 \mathrm{rpm}$ and the DMR and DIRBE beams therefore scan the range from $64^{\circ}$ to $124^{\circ}$ from the Sun.

The COBE design evolved significantly since it was first proposed in 1974. The initial concept was proposed by a Goddard-MIT-Princeton team, while versions with only microwave radiometers were proposed by teams at JPL and Berkeley. In 1976 NASA formed a science team of 6 people, drawn from all three groups, and selected Goddard to support the mission. The science team eventually grew to include 20 members, and the Goddard effort required about 1500 civil servants and contractors. The initial concept called for launch on a Delta rocket, but in the late 1970's NASA decided to launch all payloads on the Shuttle. Following the Challenger explosion in 1986, the COBE was redesigned for launch on the Delta. To achieve this, its weight was cut in half, but no compromise in performance and few changes were needed in the scientific instruments. Only three years after the redesign was approved, the COBE was launched. Its liquid helium lasted for 10 months, but the DMR and the short wavelength channels of the 
DIRBE continued operation until January 1994. The COBE now serves for satellite communication tests.

\section{FIRAS}

\subsection{FIRAS INSTRUMENT}

The COBE FIRAS (Far Infrared Absolute Spectrophotometer) was designed to compare the CMBR spectrum to a blackbody with great precision. The most recent results show that the CMBR deviates from blackbody form by less than $0.03 \%$ of the peak intensity over the wavelength range from 0.5 to $5 \mathrm{~mm}$ (Mather et al. 1994). Our interpretation of these results has also been given (Wright et al. 1994). The FIRAS also showed that the cosmic dipole has the expected shape of the derivative of the Planck function with respect to temperature (Fixsen et al. 1994a). The primary basis of the comparison is a full beam external blackbody calibrator that can be adjusted to match the temperature of the sky. This calibration is described in Fixsen et al. (1994b). The FIRAS is the first instrument to have this opportunity in the protected space environment. The instrument is designed with multiple modes of operation and multiple detectors to test for possible systematic errors.

The instrument is a polarizing Michelson interferometer used as a spectrometer (Mather, Shafer, and Fixsen 1994): It has a beamwidth of $7^{\circ}$, defined by a compound parabolic concentrator. It is symmetrical, with differential inputs and differential outputs. It is a two-beam interferometer, whose output signal (called an interferogram) depends on the path difference between the two beams. A monochromatic input produces an output proportional to the cosine of the phase difference between the two beams. A general input produces an interferogram which is the Fourier transform of the input spectrum. These interferograms are detected, amplified, filtered, digitized, filtered and averaged again, and transmitted to the computers on the ground. They are then Fourier transformed numerically and calibrated to determine the input spectra. The mirror mechanism can scan at two different scan speeds and over two different stroke lengths, to obtain low and high spectral resolution.

The FIRAS was used to map almost the entire sky. To derive cosmological results, two terms were subtracted from the spectrum of each pixel. A dipole term compensated for the Doppler shift of the spectrum, due to the motion of the Earth. The mean spectrum of the dust in the Galaxy was also derived from the map, and a fraction of this mean spectrum was fitted to each pixel spectrum and subtracted. The resulting sky map showed almost no trace of the strong Galactic emission, so that a single dust brightness map times a single characteristic spectrum is a very good representation of 
the far infrared Galaxy.

To quantify the distortion of the mean spectrum of the whole sky, it was fitted to a sum of additional terms. The first two terms are a Planck function for the mean temperature, and a temperature adjustment times the derivative of the Planck function with respect to temperature. The second term just allows for the possibility that the first may not be calculated for the correct mean temperature, since we do not know it a priori. The third term is a small number times the function $g(\nu)$ that represents the mean Galactic spectrum. This allows for the possibility that the initial subtraction done on each pixel might have been incomplete or biased. The fourth term represents the cosmic distortion. There are two interesting forms for this: a $\mu$ form and a $y$ form (Zeld́ovich \& Sunyaev, 1969, 1970). The $\mu$ form is a chemical potential distortion, that might arise between the redshifts of about $3 \times 10^{6}$ and $10^{5}$. The second form is a Comptonization form that could arise after $z=10^{5}$.

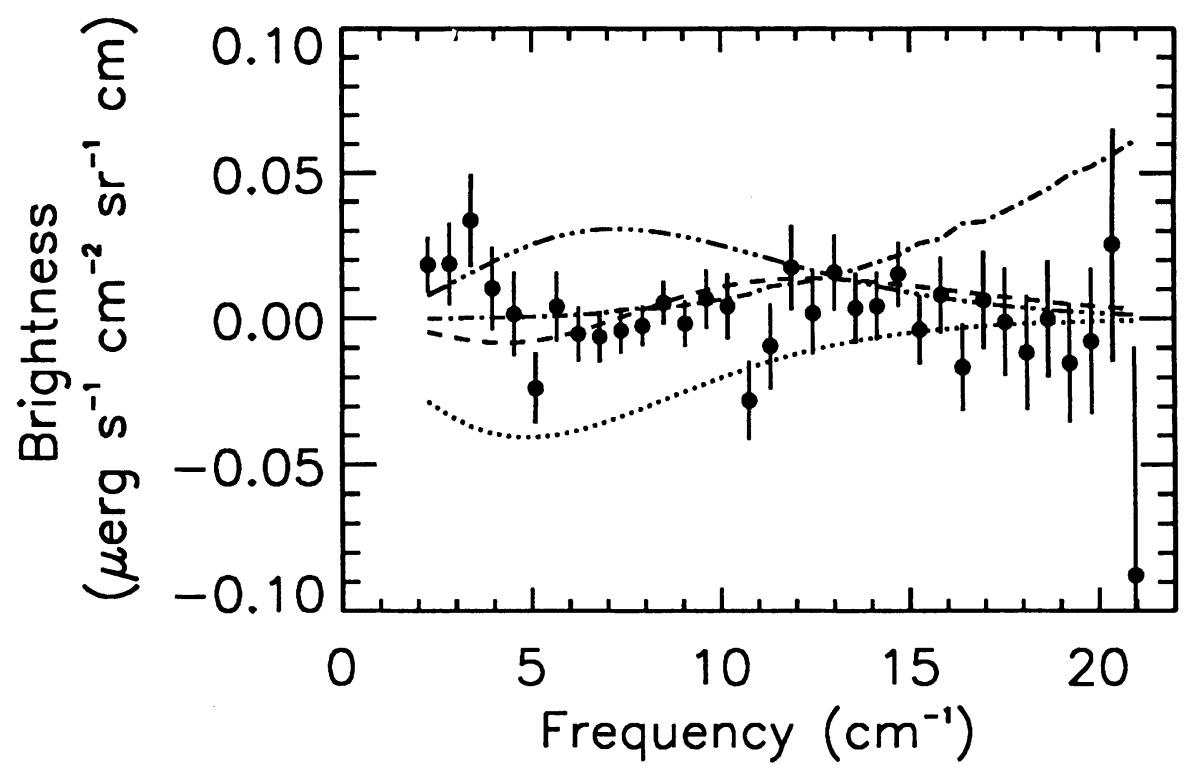

Figure 1. FIRAS measured CMBR residuals, $I_{0}-B_{\nu}\left(T_{0}\right)-\Delta T\left(\partial B_{\nu} / \partial T\right)-G_{0} g(\nu)$. Spectrum model components: the maximum allowed distortions $\left(95 \%\right.$ CL) $y=2.5 \times 10^{-5}$ $(--$,$) and |\mu|<3.3 \times 10^{-4}(\cdots)$; the Galaxy spectrum $g(\nu)$ scaled to one fourth the flux at the Galactic pole $(\cdot-)$, and the effects of a $200 \mu \mathrm{K}$ temperature shift in $T_{0}$, $0.0002\left(\partial B_{\nu}(T) / \partial T\right),(\cdots-)$. (Mather et al. 1994)

Figure 1 (Mather et al. 1994) shows the results of these least squares 
fits. The residuals are for the case where $y=\mu=0$ and show that the CMBR spectrum is the same as a blackbody within $0.03 \%$ of the peak intensity. The weighted rms residual is only $0.01 \%$ of the peak brightness. Nevertheless, these residuals are about twice as large as those expected from detector noise alone. We do not presently know the cause of this but have arbitrarily increased the size of the error bars throughout. The $y$ and $\boldsymbol{\mu}$ curves show the shapes of distortion that would be produced if these coefficients have the $95 \%$ confidence limit values of $|y|=2.5 \times 10^{-5}$ and $|\mu|=3.3 \times 10^{-4}$. These limits allow for the increased uncertainty noted above, and for variations of the derived $y$ and $\mu$ as the boundary of the selected data set moves farther from the Galactic plane.

Other possible distortions can also be explored. As an example, if the CMBR spectrum is a graybody, then we can limit its emissivity to $1 \pm$ $0.00041(95 \% \mathrm{CL})$. If the true cosmic signal contains a distortion with the same shape as the Galactic signal, then we cannot determine it with this method alone. Similarly, a cosmic energy release that simply changed the CMBR temperature could not be recognized.

The temperature of the CMBR is also important, although few cosmological calculations require a more precise number. The measured temperature is primarily needed for comparisons with different experiments, such as ground-based measurements and interstellar CN measurements. The FIRAS has two ways to determine this number. First, the thermometers in the external calibrator are the primary scale. When the calibrator is set to match the sky brightness, its thermometers measure the sky temperature. This method gives $2.730 \mathrm{~K}$. The second method uses the wavelength scale of the FIRAS to determine color temperatures. In effect, we measure the frequency of the peak brightness of the CMBR and use fundamental constants to compute a temperature. The wavelength scale was determined from FIRAS observations of interstellar [C II] and confirmed with other lines of [N II], [C I], and CO. The color temperature method yields a CMBR temperature of $2.722 \mathrm{~K}$. These numbers differ by much more than the random errors, which are only a few $\mu \mathrm{K}$. We have no obvious explanation for the difference and simply take the mean, obtaining $\mathrm{T}=2.726 \pm 0.010 \mathrm{~K}(95 \%$ confidence). Clearly this is not a statistical determination of the error bar, which is entirely systematic error.

Fortunately, there are other confirmations that this number is approximately correct. A sounding rocket experiment launched only weeks after the COBE by Gush, Halpern, and Wishnow (1990) with a similar instrument and greatly superior detectors obtained the result $2.736 \pm 0.017 \mathrm{~K}$. Interstellar CN results are also in agreement with these numbers (Roth, Meyer, \& Hawkins 1993, and Kaiser \& Wright 1990). We can also determine the temperature from the spectrum of the dipole anisotropy. This can be done 
from both DMR and FIRAS data. The FIRAS result is determined only from the color temperature, since the absolute value of the Earth's velocity is not known. The FIRAS yields $2.714 \pm 0.022 \mathrm{~K}(95 \% \mathrm{CL})$. The DMR result (see below) is $2.76 \pm 0.18 \mathrm{~K}$, and is derived from the known variation of the Earth's velocity around the Sun. The spectrum of the FIRAS dipole was fit to an amplitude of $3.343 \mathrm{mK}$. The dipole residuals are very small and are close to the value expected from detector noise alone.

\subsection{FIRAS INTERPRETATION}

Our interpretation of these spectrum distortions is given by Wright et al. (1994) and summarized here. Large CMBR spectrum distortions are very difficult to produce in plausible versions of the hot Big Bang universe. After the annihilation of positrons, the CMBR energy density far exceeded even the rest mass energy density of the baryonic matter until quite recently. Consequently, there are few processes involving the baryonic matter that can liberate much energy and change the CMBR spectrum significantly. It is even more difficult to produce enough energy to create the whole CMBR radiation from anything except a hot Big Bang.

Therefore, the most immediate conclusion of the FIRAS spectrum distortion measurements is that the hot Big Bang is the only natural explanation for a nearly perfect blackbody. Carefully tailored models are required if one desires to produce the whole energy content of a blackbody spectrum by adding up non-blackbody contributions at different redshifts, as required by alternatives to the hot Big Bang. If one imagines that dust in intergalactic space can thermalize the radiation, then that dust must have substantial optical depth over an interval of cosmic history. That moment cannot be recent, or we would not be able to see distant galaxies at far infrared wavelengths. The IRAS galaxy at $z=2.286$ demonstrates that one can see very far, and if the millimeter wave optical depth were large we would not see even such a spectacular object.

The next conclusion is that rather little of the energy of the CMBR was added to it after the first year of the expansion. The fraction of the CMBR energy added is approximately $0.71 \mu$ in the redshift range $3 \times 10^{6}>z>10^{5}$. For later redshifts, the fraction is $4 y$. A more precise calculation gives the results shown in Fig. 2.

There are many possible sources of such energy conversions, including decay of primeval turbulence, elementary particles, cosmic strings, or black holes. The growth of black holes, quasars, galaxies, clusters, and superclusters might also convert energy from other forms. The FIRAS data, together with the DMR anisotropy measurements, provide a limit on the spectral index of primordial density fluctuations. Wright et al. (1994) found an upper 


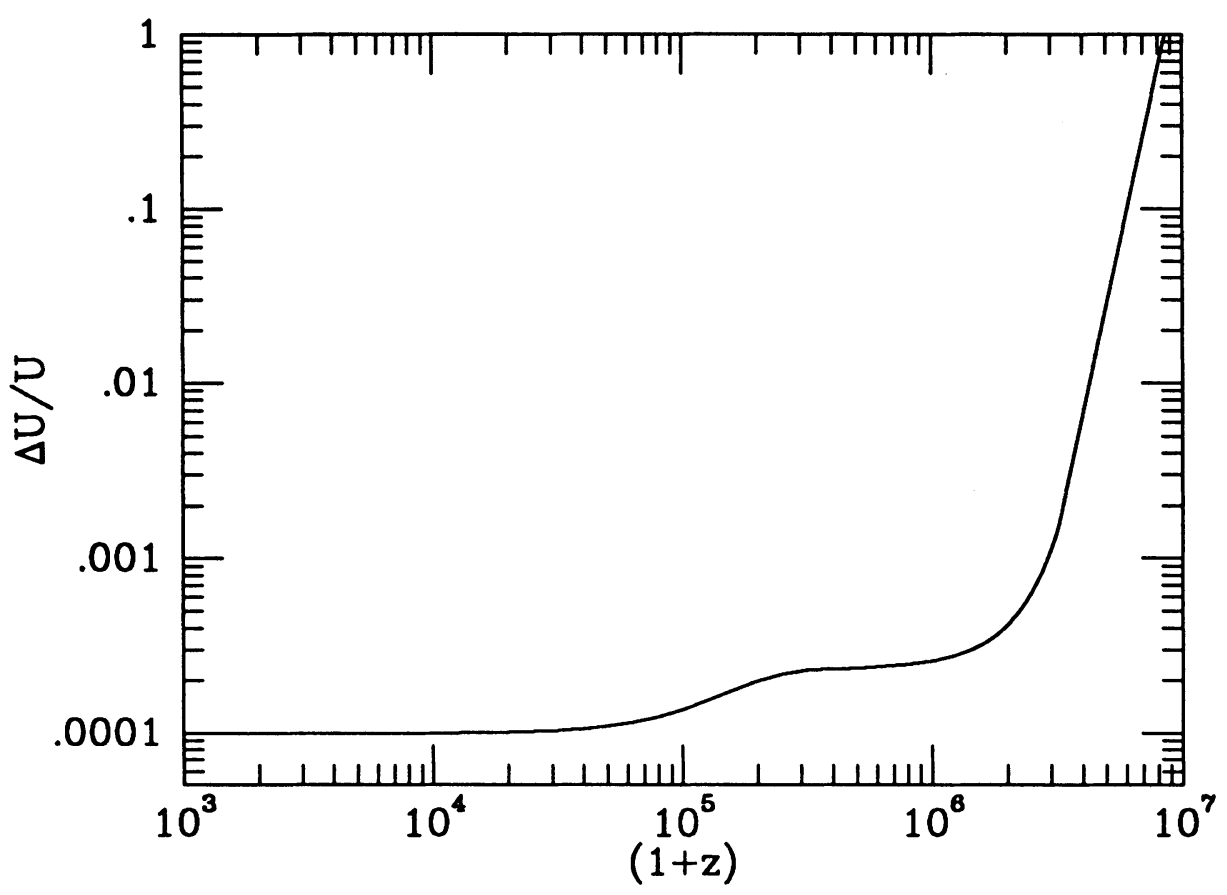

Figure 2. Limits on Energy Release Prior to Recombination (Wright et al. 1994)

limit of 1.9, while Hu, Scott, and Silk (1994) claim an upper limit of about 1.55. It is interesting that these calculations give tighter limits than existing direct measurements, even though the FIRAS numbers are a non-detection. These results are dependent on assuming that a power law is the correct form for the fluctuations over 7 orders of magnitude of scale. There is little possibility of observational evidence to confirm this assumption over such a wide range, since small scale fluctuations have long since been replaced by nonlinear phenomena.

Wright et al. (1994) also give limits on hydrogen burning following the decoupling. These results depend on using geometrical arguments (a csc $|b|$ fit) to estimate the maximum amount of extragalactic energy that could have a spectrum similar to that of our own Galactic dust. We found a limit that is a factor of about 3 smaller than the polar brightness of the Milky Way. A better understanding of the Galactic dust would help produce a tighter limit on these extragalactic signals.

Consider first population III stars liberating energy that is converted 
by dust into far infrared light (using an optical depth of 0.02 per Hubble radius), and assume that $\Omega_{b} h^{2}=0.015$. In that case less than $0.6 \%$ of the hydrogen could have been burned after $z=80$. As a second example, consider evolving infrared galaxies as observed by the IRAS. For reasonable assumptions, we found that less than $0.8 \%$ of the hydrogen could have been burned in evolving IR galaxies.

We also obtained limits on the heating and reionization of the intergalactic medium. It does not take very much energy to reionize the medium, relative to the CMBR energy, because there are so few baryons relative to CMBR photons. Even the strict FIRAS limits permit a single reionization event to occur as recently as $z=5$. More detailed calculations by Durrer (1993) show that the energy required to keep the intergalactic medium ionized over long periods of time is much more substantial and quite strict limits can be obtained. If the FIRAS limits were about a factor of 5 more strict, then it would be possible to test the ionization state of the IGM all the way back to the decoupling.

If the IGM were hot and dense enough to emit the diffuse $\mathrm{X}$-ray background light, it should distort the spectrum of the CMBR by inverse Compton scattering. This is a special case of the Comptonization process, with small optical depth and possibly relativistic particles. Calculations show that a smooth hot IGM could have produced less than $10^{-4}$ of the X-ray background, and that the electrons that do produce the $\mathrm{X}$-ray background must also have a filling factor of less than $10^{-4}$.

\subsection{INFRARED ASTRONOMY WITH FIRAS}

The FIRAS produced the first nearly all-sky far IR survey of Galactic emission at wavelengths greater than $120 \mu \mathrm{m}$ (Wright et al. 1991). The general features seen in the maps are very similar to those found by the IRAS satellite at 60 and $100 \mu \mathrm{m}$. Dust is concentrated in the Galactic plane and is heated by starlight to a temperature ranging from 15 to $30 \mathrm{~K}$, depending on the intensity of the starlight incident on the dust. This dust controls radiative transfer of energy through the Galaxy, and thus the heating and cooling of the interstellar gas. The dust has a mean spectrum which is approximated well by the form $\nu^{\alpha} B_{\nu}(T)$, where the index $\alpha=1.65$ and $T=23.3 \mathrm{~K}$. The mean dust spectrum of the Galaxy can also be well represented by the sum of two such terms with the index $\alpha=2$, with cold dust with a temperature of about $4.8 \mathrm{~K}$ and warmer dust with a temperature of $20.4 \mathrm{~K}$. In this representation, the optical depth of the cold dust is 6.7 times that of the warmer dust component. There are many possible explanations for this kind of dust spectrum. It is possible that there are multiple types of dust grains, so that some can have a very different equilibrium temperature 
than others. Dust grains could have unusual shapes, like needles (Wright 1982) or fractals (Wright 1987). Small grains with low heat capacities could have variable temperatures, as they are excited by discrete UV photons or cosmic rays. Some dust is protected within optically thick clouds and must reach much lower temperatures than that exposed to the full intensity of the interstellar radiation field, and other dust is very close to stars and must be much warmer than average. The total dust luminosity of the Galaxy is $(1.8 \pm 0.6) \times 10^{10} \mathrm{~L}_{\odot}($ Wright et al. 1991).

The FIRAS also provided the first panoramic and unbiased spectral survey of the far IR line emissions from the interstellar medium, including $\mathrm{CO},[\mathrm{C} \mathrm{I}],[\mathrm{C} \mathrm{II}]$, and [N II] lines. These results were reported by Wright et al. (1991) and by Bennett et al. (1994). The FIRAS map of the emission of [C II] at $158 \mu \mathrm{m}$ shows it to be extremely widespread, both in and out of the Galactic plane. This line is the dominant cooling mechanism for most of the interstellar gas, and is the brightest emission line of the Galaxy, accounting for $0.3 \%$ of the dust luminosity. The strength of the line is so great that it can be seen by eye in the raw interferograms as an undamped sinusoid, and with more analysis the Galactic rotation curve can be seen in the line center frequency. The [C II] line is very strongly correlated with the dust emission, as expected from the theory of photodissociation regions. The heating of the gas is provided mostly by photoelectrons emitted from dust grains, and must be in balance with the cooling provided mostly by the [C II] line. Deviations from this correlation are expected where the dust properties change, where the interstellar radiation field changes color, or where other heating and cooling mechanisms become important at extremes of temperature or density. In particular, the ratio of [C II] to dust decreases to nearly zero at the Galactic poles.

The FIRAS also detected the emission of ionized nitrogen [N II] at 205 $\mu \mathrm{m}$ for the first time and measured its frequency well enough for further astronomical (Petuchowski and Bennett 1992) and laboratory (Brown et al. 1994) observations. This is the second brightest far IR line from the interstellar gas and probes a different phase of the interstellar medium because nitrogen ionizes less easily than hydrogen and carbon. The nitrogen and carbon line intensities are also strongly correlated but show the relation $[\mathrm{N} \mathrm{II}] \propto[\mathrm{C} \mathrm{II}]^{3 / 2}$. The meaning of this correlation was explored by Petuchowski and Bennett (1993).

Carbon monoxide is the standard tracer for interstellar molecular gas. The FIRAS has mapped the brightness of the lines of CO from the 2-1 up to the 6-5 transitions, showing that the line ratios are highly dependent on location in the Galaxy. In particular, the $\mathrm{CO}$ lines are extremely bright relative to the others in the Galactic Center, where the gas temperature and density are very high and the UV field is shielded by dust. The two 
lines of neutral carbon [C I] are also seen and mapped by the FIRAS.

Weaker lines observed by the FIRAS include oxygen and water. The water line at $269 \mu \mathrm{m}$ has never been observed before in astrophysical sources because of the extremely high optical depth of the terrestrial absorption line. The line is seen in absorption by FIRAS (Bennett et al. 1994) against the continuum emission of the Galactic Center, but at a low level of significance relative to the detector noise and possible systematic errors. The observed absorption is comparable to that expected from the concentration of interstellar water vapor.

\section{Data Delivery}

A set of initial data products from the Cosmic Background Explorer Satellite is now available for analysis, and further data releases are planned for summer of 1994. A COBE Guest Investigator Facility, which supports proposers funded under NASA's Astrophysics Data Program, has been established at the Goddard Space Flight Center. The data are distributed from the National Space Science Data Center (NSSDC). All of the initial data products are available via anonymous ftp and accessible on the World Wide Web; most of the datasets larger than about $10 \mathrm{MB}$ will be distributed on a set of CD-ROMs.

The data now available, and the associated documentation, may be obtained by anonymous ftp to nssdca.gsfc.nasa.gov (equivalent IP address 128.183.36.23). Please provide your e-mail address as the password. Change to directory ( cd) anon_dir:[000000.cobe] ("cd cobe" should work) and download the file called AAREADME.DOC for further instructions. Alternatively, the data and documentation may be obtained on tape by request to the Coordinated Request and User Support Office (CRUSO), NASA Goddard Space Flight Center, Code 633.4, Greenbelt, MD 20771. They may be requested by phone at 301-286-6695, or by e-mail from request@nssdca.gsfc.nasa.gov. For assistance in the use of COBE data, contact David Leisawitz by phone (301-286-0807), FAX (301-286-1771), e-mail ( leisawitz@stars.gsfc.nasa.gov), or surface mail at the address given above. Please address requests for preprints or reprints of COBE Team publications to Susan Adams as follows: Ms. Susan Adams, NASA Goddard Space Flight Center, Code 685, Greenbelt, MD 20771, phone: 301-286-4257, or FAX: 301-286-1617, or e-mail: adams@stars.gsfc.nasa.gov. The COBE data are accessible on the World Wide Web at the following Uniform Resource Locator (URL) address:

http://ww .gsfc.nasa.gov/astro/cobe_home.html 


\section{Summary and Conclusions}

The COBE was designed to answer three questions: what is the spectrum of the CMBR, what are its anisotropies, and what is the brightness of the CIBR? The first two have been answered. The CMBR spectrum is very close to a blackbody of $\mathrm{T}=2.726 \mathrm{~K}$, and the weighted rms deviation from the blackbody form is less than $0.01 \%$. The CMBR has anisotropies on all size scales larger than $7^{\circ}$, with a size spectrum consistent with the predicted scale-invariant primordial fluctuations. They have an amplitude of 11 parts per million on a $10^{\circ}$ scale. The CIBR has not yet been found, but the data are in hand and analysis is in progress. All three instruments have given new views of the Galaxy, showing large scale features due to the interstellar medium and the general distribution of material. The Galactic bulge at the center is revealed at wavelengths where it is less obscured by dust absorption, and the Galaxy appears to have a central bar. Line emission from neutral and ionized carbon, ionized nitrogen, and carbon monoxide are widespread, and water may have been seen in absorption against the Galactic Center. The interplanetary dust (zodiacal light) has been observed more extensively than ever before, at a wide range of elongations from the Sun and throughout the year, enabling a new and more complete understanding of the dynamics and sources of the cloud.

\section{Acknowledgements}

The National Aeronautics and Space Administration/Goddard Space Flight Center (NASA /GSFC) is responsible for the design, development, and operation of the Cosmic Background Explorer (COBE). GSFC is also responsible for the software development and the final processing of the mission data. The COBE SWG is responsible for the definition, integrity, and delivery of the public data products, and includes the following members:

NAME

J. C. Mather

M. G. Hauser

G. F. Smoot

C. L. Bennett

N. W. Boggess

E. S. Cheng

E. Dwek

S. Gulkis

\section{AFFILIATION SPECIAL ROLE}

GSFC

GSFC

UC Berkeley

GSFC

GSFC-ret

GSFC

GSFC

JPL
Project Scientist and FIRAS Principal Investigator DIRBE Principal Investigator DMR Principal Investigator

DMR Deputy PI

Deputy Proj. Scientist

Deputy Proj. Scientist 
M. A. Janssen JPL

T. Kelsall

P. M. Lubin

S. S. Meyer

S. H. Moseley

T. L. Murdock

R. A. Shafer

R. E. Silverberg

J. Vrtilek

R. Weiss

D. T. Wilkinson

E. L. Wright
GSFC

UCSB

MIT

GSFC

Gen.Res.Corp.

GSFC

GSFC

NASA HQ

MIT

Princeton

UCLA
DIRBE Deputy PI

FIRAS Deputy PI

Program Scientist

Chairman of SWG

Data Team Leader

Many people have made essential contributions to the success of COBE in all its stages, from conception and approval through hardware and software development, launch, and flight operations. To all these people, in government agencies, universities, and industry, the science team and I extend my thanks and gratitude. In particular, I thank the large number of people at the GSFC who brought this challenging in-house project to fruition.

\section{References}

Barney, R. D. (1991) Iluminating Eng. Soc. J., 34, 34

Bennett, C. L. (1991) IAU XXI Highlights Astron, J. Bergeron, Ed., 9, 335

Bennett, C. L. et al. (1992) ApJ, 391, 466

Bennett, C. L. et al. (1994) ApJ, 434, 587-598

Boggess, N. W. "The Cosmic Background Explorer (COBE): The Mission and Science Overview," (1991) IAU XXI Highlights Astron, J. Bergeron, Ed., 8, 273

Boggess, N. W. et al. (1992) $A p J, 397,420$

Bromberg, B. W. \& Croft, J. (1985) Adv. Astron. Sci., 57, 217

Brown, J. M. et al. (1994) $A p J L, 428$, L37

Coladonato, R. J. et al. (1990) Proc. Third Air Force/NASA Symp. on Recent Advances in Multidisciplinary Analysis and Optimization, 370, Anamet, Hayward, CA

Durrer, R. (1993) "Early Reionization in Cosmology," Infrared Phys. Technol., 35, 83-94

Fixsen, D. J. et al. (1994a) ApJ, 420, 445-449

Fixsen, D. J. et al. (1994b) $A p J, 420,457-473$

Gulkis, S., Lubin, P. M., Meyer, S. S., \& Silverberg, R. F. (1990) Sci. Amer., 262, 132

Gush, H.P., Halpern, M., \& Wishnow, E.H. (1990) Phys. Rev. Lett., 65, 537

Hauser, M. G. et al. (1991) After the First Three Minutes, AIP Conf. Proc, 222, 161, eds. S. S. Holt, C. L. Bennett, \& V. Trimble, New York

Hauser, M. G. (1991) IAU XXI Highlights Astron, J. Bergeron, Ed., 8, 291

Hawkins, I., and Wright, E. (1988) $A p J, 324,46-59$

Hopkins, R. A., \& Castles, S. H. (1985) Proc. SPIE, 509, 207

Hopkins, R. A., \& Payne, D. A. (1987) Adv. Cryogenic Engineering, 33, 925

Hu, W., Scott, D., and Silk, J. (1994), "Power Spectrum Constraints from Spectral Distortions in the Cosmic Microwave Background," ApJ, 430, L5 
Janssen, M. A. \& Gulkis, S. (1991) Proc. The Infrared and Submillimetre Sky After COBE, Les Houches, 391, ed. M. Signore \& C. Dupraz, Kluwer, Dordrecht

Kaiser, M. E., and Wright, E. L. (1990) $A p J, 356, \mathrm{~L} 1$

Mather, J. C. (1982) Opt. Eng., 21, 769

Mather, J. C. et al. (1990) IAU Colloq. 123, Observatories in Earth Orbit and Beyond, Proc., ed. Y. Kondo, 9, Kluwer, Boston

Mather, J. C. et al. (1991a) AIP Conf. Proc. After the First Three Minutes, 222, 43, ed. S. S. Holt, C L. Bennett, \& V. Trimble, AIP, New York

Mather, J. C. (1991b) IAU XXI Highlights Astron, J. Bergeron, Ed., 9, 275

Mather, J. C., Shafer, R.A., and Fixsen, D. J. (1993) Proc. SPIE, 2019, 146-157

Mather, J. C. et al. (1994) ApJ, 420, 439-444

Milam, L. J. (1991) Mluminating Eng. Soc. J., 34, 27

Mosier, C. L. (1991) AIAA 29th Aerospace Sciences Conference, 91-361

Petuchowski, S. J. \& Bennett, C. L. (1992) ApJ, 391, 137-140

Petuchowski, S. J. \& Bennett, C. L. (1993) ApJ, 405, 595-598

Roth, K. C., Meyer, D., \& Hawkins, I. (1993) ApJL, 413, L67-L71

Sampler, H. P. (1990) Proc. SPIE, 1340, 417

Smoot, G. F. (1991) IAU XXI Highlights Astron, J. Bergeron, Ed., 8, 281

Smoot, G. F. et al. (1991) ApJ, 371, L1

Volz, S. M. \& DiPirro, M. J. (1992) Cryogenics, 32, 77

Volz, S. M. \& Ryschkewitsch, M. G. (1990) Superfluid Helium Heat Transfer, HTD, 134, 23, ed. J. P. Kelly \& W. J. Schneider AME, New York

Volz, S. M., Dipirro, M. J., Castles, S. H., Rhee, M. S., Ryschkewitsch, M. G., \& Hopkins, R. (1990) Proc. Internat. Symp. Optical and Opto-electronic Applied Sci. and Eng., 268, SPIE, San Diego

Volz, S. M., Dipirro, M. J., Castles, S. H., Ryschkewitsch, M. G., \& Hopkins, R. (1991) Adv. Cryogenic Engineering, 37A, 1183

Wright, E. L. (1982) ApJ, 255, 401-407

Wright, E. L. (1991) Proc. The Infrared and Submillimetre Sky After COBE, Les Houches, 231, ed M. Signore \& C. Dupraz, Kluwer, Dordrecht

Wright, E. L. (1987) $A p J$, 320, 818-824

Wright, E. L. (1990) Ann. NY Acad. Sci., Proc. Texas-ESO-CERN Sym, 647, 190

Wright, E. L. et al. (1991) ApJ, 381, 200

Wright, E. L. et al. (1994) ApJ, 420, 450-456

Zeld́ovich, Ya. B., and Sunyaev, R.A. (1969) Ap\&SS, 4, 301

Zeld́ovich, Ya. B., and Sunyaev, R.A. (1970) Ap\&SS, 7, 20 